\title{
Von der religiösen zur interreligiösen Bildung? Einwände, theoretische Klärungen und empirische Befunde zur Wirksamkeit
}

\author{
Friedrich Schweitzer
}

Eingegangen: 16. März 2021 / Überarbeitet: 23. August 2021 / Angenommen: 8. Oktober 2021 / Online publiziert: 25. Februar 2022

(C) Der/die Autor(en) 2022

Zusammenfassung Ausgangspunkt dieses Beitrags ist die in Öffentlichkeit und Politik verbreitete Forderung nach einer Erweiterung der religiösen zur interreligiösen Bildung. Darüber hinaus werden Einwände gegen ein solches Programm aufgenommen, die eine „Religiösierung“ sozialer Probleme sowie ein „Othering“ befürchten. Anhand theoretischer Klärungen zu interreligiöser Bildung werden diese Einwände diskutiert, vor allem aber mit der Frage nach der Wirksamkeit interreligiöser Bildung verbunden. Dazu wird der Stand der Forschung hinsichtlich unterschiedlicher Konzeptualisierungen sowie der Befundlage dargestellt sowie exemplarisch eine Studie zum „Interreligiösen Lernen durch Perspektivenübernahme“ vorgestellt. Die Befunde belegen eine teilweise Wirksamkeit interreligiöser Bildung, verweisen aber zugleich auf ausbleibende Effekte bei Einstellungen sowie auf einen Bedarf didaktischer Präzisierungen. Die Konzeptualisierungen belegen die Möglichkeit, interreligiöse Bildung nicht im Sinne einer Verfestigung religiöser Identitäten oder einer Selbstabschließung zu verstehen, sondern einer wachsenden Vertrautheit mit anderen Religionen und der Offenheit für Überzeugungen, die sich von den eigenen unterscheiden.

Schlüsselwörter Interreligiöse Bildung · Othering · Perspektivübernahme

Prof. Dr. Dr. h.c. Friedrich Schweitzer $(\triangle)$

Evangelisch-Theologische Fakultät, Universität Tübingen, Liebermeisterstr. 12, 72076 Tübingen,

Deutschland

E-Mail: Friedrich.Schweitzer@uni-tuebingen.de 


\title{
From religious to inter-religious education? Objections, theoretical clarifications and empirical results concerning the effectiveness of inter- religious approaches
}

\begin{abstract}
This article makes the plea for broadening religious education to interreligious education its starting point. While this plea may often be found in public debates and in politics there have also been objections to respective programs which are based on fears of "religionism" and effects of religious "othering". These objections are discussed theoretically but most of all in connection with the question of the effectiveness of inter-religious education. The state of the art of respective research is summarized, including different conceptual models as well research results and, as an example, an empirical study on "inter-religious education through perspective-taking". The results show that interreligious education is, in part, effective but that there are no effects on pupils' attitudes. Moreover, there seems to be a need for further refinements concerning methods and teaching designs. The conceptual models indicate that it is possible to conceptualize inter-religious education such that entrenched or self-absorbed religious identities are avoided while supporting familiarity with other religions as well as strengthening openness for religious convictions different from one's own.
\end{abstract}

Keywords Inter-religious education - Empirical research on effectiveness · Othering $\cdot$ Perspective-taking

Die Erwartung, dass sich religiöse Bildung unter heutigen Voraussetzungen zur interreligiösen Bildung weiterentwickeln müsse, ist inzwischen weit verbreitet. Sie richtet sich nicht nur auf das Schulfach Religion und seine Äquivalente, sondern betrifft alle Formen der religionsbezogenen Bildung innerhalb und außerhalb der Schule. Der Wandel der Gesellschaft im Sinne zunehmender Multikulturalität und Multireligiosität verweist auf neue pädagogische Aufgaben, die es vordringlich machen, den traditionellen Umkreis einer Erziehung oder Bildung, die sich nur auf eine - in einem Land vorherrschende oder der eigenen Herkunft entsprechende Religion bezieht, zu überschreiten. Ein Zusammenleben in Frieden und Toleranz, wechselseitigem Respekt und Anerkennung scheint ohne eine auch pädagogische Vorbereitung von Kindheit an kaum zu erreichen.

Auch in der Praxis der Schule geschieht bereits vieles, was der interreligiösen Bildung dienen soll. In Bildungsplänen hat dies ebenso seinen Niederschlag gefunden wie in Schulbüchern und Unterrichtsmaterialien, in der religionspädagogischtheoretischen Literatur ebenso wie in kirchlichen Stellungnahmen. Die Rede von interreligiöser Bildung statt von interreligiösem Lernen soll dabei einen grundlegenden Bildungsanspruch für diesen Bereich zum Ausdruck bringen, der über einzelne Lernformen oder -inhalte hinausgeht (vgl. Schweitzer 2014).

Zugleich fällt allerdings auf, dass interreligiöse Lern- oder Bildungsaufgaben in der Erziehungswissenschaft bislang wenig Aufmerksamkeit erfahren. Auch wenn dies teilweise auf die generelle religiöse Abstinenz dieser Disziplin sowie auf die sog. „Verdrängung“ von Religion in der Erziehungswissenschaft zurückzuführen 
sein mag (vgl. Oelkers et al. 2003), gibt es zugleich spezifische Gründe, die aus einem in der Erziehungswissenschaft verbreiteten Kultur- und Religionsverständnis resultieren oder, noch weiterreichend, aus der expliziten Auffassung, dass kulturoder religionsbezogene Deutungen etwa von gesellschaftlichen Problemen im Zusammenleben prinzipiell problematisiert oder abgelehnt werden müssten. In dieser Sicht blenden auf interreligiöse Verhältnisse bezogene Versuche, gesellschaftliche Konflikte zu erklären oder zu ihrer Bewältigung beizutragen, die tatsächlichen - sozialen, politischen und materiellen - Konfliktursachen aus. Wo dies nicht beachtet wird, sei von „Religiösierung“ zu sprechen und häufig auch vor einem „Othering“, bei dem Kindern und Jugendlichen pädagogisch von außen eine religiöse Identität auferlegt wird (vgl. Lingen-Ali und Mecheril 2016; s. auch Radtke 2011 sowie Diehm 2010; Hamburger 2009).

Damit steht der geforderte Übergang von der religiösen zur interreligiösen Bildung zunächst vor einer Plausibilisierungsaufgabe, gerade im Verhältnis zur Erziehungswissenschaft. Darüber hinaus stellt sich aber die Frage, wie es um die tatsächliche Wirksamkeit interreligiöser Bildung steht. Aus der Forderung, dass pädagogisch bestimmte Aufgaben übernommen werden müssten, folgt bekanntlich keineswegs, dass dies auch erfolgreich geschehen kann. Auch in der Religionspädagogik ist gelegentlich zu hören, dass interreligiöse Bildung im Rahmen von Schule und Unterricht nicht möglich sei (vgl. Dressler 2003).

Für den vorliegenden Beitrag ergibt sich aus dieser Diskussionslage eine doppelte Aufgabe: Zunächst sind die erziehungswissenschaftlichen Einwände gegen ein Bildungsverständnis, das die interreligiöse Dimension hervorhebt, zu diskutieren. Dies soll hier allerdings nicht in erster Linie in theoretischer Hinsicht geschehen, sondern in Verbindung mit der zweiten Aufgabe, nämlich der Prüfung der Wirksamkeit entsprechender Bildungsangebote. Die entsprechende Frage kann dann folgendermaßen gefasst werden: Kann es gelingen, interreligiöse Bildungsangebote so zu konzeptualisieren, dass sie weder dem Vorwurf einer Religiösierung noch eines Othering ausgesetzt sind und sich zugleich als wirksam erweisen? Dazu sollen die wichtigsten empirischen Untersuchungen aus diesem Bereich aufgenommen und soll ein Forschungsprojekt ,interreligiöses Lernen durch Perspektivenübernahme“ dargestellt werden. Vorab muss allerdings genauer geklärt werden, was unter interreligiöser Bildung verstanden werden kann. Am Ende soll versucht werden, die theoretischen Perspektiven und empirischen Befunde aufeinander zu beziehen und Aufgaben für die Weiterarbeit zu beschreiben.

\section{Begründungen, Begriff und Ziele interreligiöser Bildung}

\subsection{Begründung interreligiöser Bildungsaufgaben}

Die wichtigste Begründung interreligiöser Bildung ergibt sich aus der gesellschaftlichen Situation der Gegenwart, die ihrerseits in einem globalen Zusammenhang gesehen werden muss. Angesichts der zunehmenden religiös-weltanschaulichen Vielfalt in der Gesellschaft wächst der Orientierungsbedarf auf gesellschaftlicher ebenso wie auf individueller Ebene. Die Ausführungen im Folgenden konzentrieren sich 
auf den Aspekt der Multireligiosität - nicht-religiöse Weltanschauungen bedürften einer eigenen Thematisierung.

In der Literatur werden im Blick auf die Multireligiosität wünschenswerte Orientierungen vielfältige Aspekte angesprochen, die ebenso das Wissen über verschiedene Religionen betreffen wie eine entsprechende Deutungsfähigkeit, aber auch Einstellungen und Werte etwa im Sinne der Offenheit für religiöse Ausdrucksund Praxisformen, die sich von den eigenen unterscheiden (vgl. u.a. Lähnemann 1998; Leimgruber 2007; Nipkow 1998 sowie die im folgenden genannte Literatur zur interreligiösen Bildung). An Dringlichkeit haben im vergangenen Jahrzehnt darauf bezogene pädagogische Aufgaben durch die Entwicklungen bei Migration und Flucht gewonnen, in deren Zusammenhang das friedliche Zusammenleben in Deutschland und Europa durch das Fehlen gemeinsamer Wertorientierungen infrage gestellt scheint. Dies war und ist beispielsweise an den wiederkehrenden Forderungen nach einer ,deutschen Leitkultur“ abzulesen, die in pädagogischer Sicht allerdings bestenfalls als Problemanzeige und nicht als wissenschaftlich begründete Deutung gelten können (vgl. Schweitzer 2014, S. $21 \mathrm{ff}$.). In diesem Falle geht es auch nicht um interreligiöse Lernprozesse, sondern um die Auffassung, dass Integration einfach die Anpassung an die Verhältnisse in Deutschland bedeuten müsse. Solche Reaktionen lassen sich ihrerseits als Mangel an interreligiöser Bildung verstehen, da es offenbar an Einsicht in die tatsächliche Dynamik migrationsbedingter Begegnungen fehlt und ebenso an Offenheit für andere Kulturen und Religionen.

Begründet werden kann die Aufgabe interreligiöser Bildung aber auch mit anderen gesellschaftlichen Tendenzen, wie sie etwa im (neuen) Antisemitismus oder der Islamfeindlichkeit zutage treten. Solche Einstellungen sind empirischen Befunden zufolge in Deutschland zunehmend verbreitet (vgl. etwa Religionsmonitor 2015). Bei einer aktuellen Repräsentativstudie in Baden-Württemberg beispielsweise waren $25 \%$ der befragten Jugendlichen der Meinung, dass es in Deutschland ,zu viele Muslime“" gebe (Schweitzer et al. 2018, S. 114).

Die Forderung nach einer interreligiösen Bildung, die auf wechselseitige Offenheit von Angehörigen verschiedener Religionen sowie auf dialogische Verhältnisse zielt, sieht sich - wie einleitend erwähnt - allerdings dem Einwand aus der Erziehungswissenschaft ausgesetzt, dass Probleme des gesellschaftlichen Zusammenlebens auch unter der Voraussetzung von Migration und religiöser Vielfalt nicht auf religiöse Ursachen zurückzuführen seien, sondern in aller Regel auf soziale Probleme und gesellschaftliche Machtverhältnisse (vgl. Radtke 2011, S. 43-58). Der Fokus auf Religion führe zu problematischen, weil einseitigen Zuspitzungen und Attributionen - abzulesen daran, dass aus „Türken“ im öffentlichen Diskurs und in den Medien mehr und mehr „Muslime“ geworden sind und Probleme des Zusammenlebens dann auf „,den Islam“ zurückgeführt werden. Machtförmig sei darüber hinaus das religiöse „Othering“, durch das der religiös Andere projektiv erst konstruiert wird (vgl. Lingen-Ali und Mecheril 2016). Auch die Vorstellung von Religionsoder Kulturdialog basiere vielfach auf Homogenisierungsprozessen, durch welche die Angehörigen einer bestimmten Religion zu einer einheitlichen Größe hypostasiert werden (,Kulturen sprechen nicht“, Radtke 2011).

Solche Einwände sind ernst zu nehmen und werden auch in der Religionspädagogik seit langem rezipiert, etwa wenn Religionismus und Religiösierungen kon- 
sequent als problematisch indiziert und deshalb ausgeschlossen werden sollen (vgl. schon Hull 1996). Doch stellt sich auch umgekehrt die Frage, ob religiöse Unterschiede am besten stillschweigend übergangen werden, so wie dies derzeit in der Erziehungswissenschaft weithin der Fall ist. Ein gutes Beispiel ist der aktuelle Heterogenitätsdiskurs, in dem auf möglichst viele Unterschiede Bezug genommen wird, in aller Regel aber eben nicht auf religiöse Unterschiede (so schon bei Prengel 1993; als aktuelles Lehrbuch Bohl et al. 2017). Gerade bei antisemitischen oder islamfeindlichen Einstellungen scheinen immer wieder neu auch religiöse Stereotype eine Rolle zu spielen, und es erscheint kaum vorstellbar, dass bei der pädagogischen Arbeit am besten kein Bezug auf ihre religiöse Dimension genommen wird.

Darüber hinaus lässt sich die im Blick auf einen „Kulturalismus“ in der interkulturellen Pädagogik entwickelte Kritik von vornherein nicht bruchlos auf Ansätze der interreligiösen Bildung übertragen. Denn während Kulturen durchweg als fluide Größen anzusehen sind und keine klaren Grenzen aufweisen, sind Religionen vielfach ausdrücklich als Gemeinschaften verfasst. Solche Gemeinschaften, wie sie besonders die christlichen Kirchen darstellen, sind zwar ebenfalls durch innere Vielfalt geprägt, aber sie kennen auch ein zumindest im Prinzip gemeinsames Bekenntnis sowie eine klar definierte Mitgliedschaft. Darüber hinaus werden in und durch solche Gemeinschaften Vertreterinnen und Vertreter benannt, denen offenbar zugetraut wird, für eine bestimmte religiöse Gemeinschaft sprechen zu können. Schon daran ist abzulesen, dass der Einwand „Kulturen sprechen nicht“" so nicht ohne weiteres auf den Bereich der Religionen übertragen werden kann.

\subsection{Begriff und Bildungsziele}

Der Begriff des interreligiösen Lernens ist in der deutschen Diskussion seit etwa den 1990er-Jahren feststellbar. Zunächst wurde bewusst, um den tentativen Charakter entsprechender Angebote hervorzuheben, von interreligiösem Lernen gesprochen (Rickers 2001), später mit umfassenderem Anspruch von interreligiöser Bildung (Schweitzer 2014). Vorbild für die Begriffsbildung war das interkulturelle Lernen, wie es in Deutschland seit den 1980er-Jahren diskutiert wird (Lehrbuch: Auernheimer 1990). Da diese Diskussion weithin keinen Bezug auf Religion oder Religionen nahm, erschien und erscheint es besonders in der Religionspädagogik erforderlich, ausdrücklich von interreligiösem Lernen zu sprechen.

Inhaltlich geht es um ein Lernen, das sich auf mehr als eine Religion bezieht. Weithin durchgesetzt hat sich ein dialogisches Verständnis, demzufolge bei diesem Lernen die Glaubensüberzeugungen und Wahrheitsansprüche nicht nur der einen (eigenen), sondern beider (oder verschiedener) Religionen aufgenommen werden sollen. Daraus ergibt sich folgende zusammenfassende Definition: „Interreligiöse Bildung ist eine Dimension von Bildung, die sich auf die Wahrnehmung eigener und anderer Religionen und ihr Verhältnis zueinander bezieht, die auf wechselseitigem Verstehen beruhende dialogische Einstellungen anstrebt und zu einem gesellschaftlichen Zusammenleben im Sinne von Frieden und Toleranz, Anerkennung des Anderen und Respekt voreinander befähigt." (Schweitzer 2014, S. 132).

Die Diskussion über Ziele der interreligiösen Bildung wird bislang wissenschaftlich durch die - evangelische, katholische und islamische - Religionspädagogik 
bestimmt (vgl. die o.g. Literatur sowie Meyer 2019; Sajak 2018; Schambeck 2013; Langenhorst 2016; internationaler Überblick: Engebretson et al. 2010) und gesellschaftlich durch (bildungs-)politische Erwartungen und Vorgaben. Auch von den Kirchen und Religionsgemeinschaften gibt es entsprechende Stellungnahmen (vgl. bspw. EKD 2014). Bildungstheoretisch gesehen müssen Bildungsziele aber immer auch von der Selbstwerdung der Kinder und Jugendlichen her bestimmt werden, die in der bildungstheoretischen Tradition überhaupt an erster Stelle steht:

- Auf individueller Ebene bieten sich Bezüge zur Ausbildung einer religiösen Identität sowie zur religiösen Orientierung an (Schweitzer 2014, S. 110 ff.). So gesehen sollen Formen der religiösen Identitätsbildung, einerseits (Selbst-)Identifikationen erlauben, andererseits aber nicht auf stereotypen Abgrenzungen oder fundamentalistischen Haltungen beruhen. In religiösen und weltanschaulich pluralen Gesellschaften ist dies wohl nur so vorstellbar, dass mit der eigenen Identitätsbildung zugleich eine Orientierung im Verhältnis zu anderen religiösen Identitäten und den mit diesen vielfach verbundenen religiösen Traditionen und Institutionen gewonnen wird.

- Gesellschaftliche Erwartungen gehen häufig vom Begriff der Integration aus, der allerdings problematisiert werden muss, wenn er lediglich die Bereitschaft zur Anpassung an die hiesigen Verhältnisse meint. Bildungspläne nicht nur für den Religionsunterricht, sondern auch im Blick auf den schulischen Bildungs- und Erziehungsauftrag insgesamt können aber durchaus auch anspruchsvollere Sichtweisen vertreten und sich darin bildungstheoretischen Perspektiven annähern. Die Befähigung dazu, in einer religiös pluralen Gesellschaft handlungs- und teilhabefähig zu werden, lässt sich auch bildungstheoretisch vertreten (vgl. Benner et al. 2011).

- Wenn sich Kirchen und Religionsgemeinschaften etwa im Blick auf die Schule an dieser Zieldiskussion beteiligen (vgl. exemplarisch EKD 2014), muss darin nicht eine zu problematisierende Einmischung gesehen werden. Auch hier ist auf die demokratisch legitimierten Formen der Vertretung von Religionsgemeinschaften nach außen, beispielsweise gegenüber dem Staat, hinzuweisen. Auf dieser Vorstellung beruht auch die im Grundgesetz getroffene Regelung zum Religionsunterricht, bei der explizit die „Religionsgemeinschaften“ als Gegenüber zum Start angesprochen sind (Art. 7,3 GG). Aus heutiger Sicht leuchtet diese Regelung wohl am stärksten unter dem Aspekt einer nicht nur staatlich, sondern auch zivilgesellschaftlich verantworteten Schule ein.

Den Kirchen und Religionsgemeinschaften liegt einerseits an einer religiösen Tradierung der eigenen Glaubensüberzeugungen, andererseits aber auch an dialogischen Verhältnissen, im Sinne einer pluralistischen und friedlichen Koexistenz verschiedener Religionen. Die dabei geforderte Toleranz wird dabei zunehmend im Sinne einer Vertrautheit mit anderen Glaubensüberzeugungen verstanden, als Voraussetzung einer starken Toleranz, die auch eine genauere Kenntnis und ein Verständnis anderer Traditionen voraussetzt (vgl. Krimmer 2013).

Übergreifend wird im Blick auf die Ziele interreligiöser Bildung von Pluralitätsfähigkeit oder Pluralismusfähigkeit gesprochen (religionspädagogisch Schweitzer 
et al. 2002; aus kirchlicher Sicht beispielsweise EKD 2014), was auch aus erziehungswissenschaftlicher Sicht bejaht werden kann (Pant 2016). Diese Fähigkeit steht gleichsam zwischen den auch in gesellschaftlicher Hinsicht problematischen Polen von Fundamentalismus auf der einen und Relativismus auf der anderen Seite. Weder eine Verabsolutierung der eigenen Überzeugungen und Wertorientierungen wäre wünschenswert noch auch die Vergleichgültigung aller darauf bezogenen Fragen oder Werte. Voraussetzung dafür ist insbesondere eine prinzipienorientierte Urteilsfähigkeit, die eine reflexive Auseinandersetzung mit religiös begründeten Geltungsansprüchen erlaubt. Zu den dafür zentralen Kriterien gehören rechtliche Maßstäbe (insbesondere Grundrechte, Menschenrechte) sowie politische Vorgaben, die im Namen der Demokratie einen gleichsam äußeren Rahmen ziehen (vgl. Nipkow 1998). Das Verhältnis der Glaubensüberzeugungen zueinander lässt sich aber zureichend nicht allein von außen bestimmen, sondern erfordert religiöse Deutungen, durch die die rechtlichen und politischen Kriterien von innen her ergänzt werden. Eben deshalb liegt hier auch eine Aufgabe religiöser Bildung, die auf eine reflektierte Einsicht in solche Deutungen oder Kriterien zielt.

Die beschriebenen erziehungswissenschaftliche Einwände treffen solche Ziele interreligiöser Bildung offenbar kaum. Denn diese Ziele sind nicht auf eine Verfestigung oder Abschottung religiöser Identitäten und Traditionen eingestellt, sondern auf dialogische Offenheit sowie dauerhaft vielfältige Verhältnisse. Diese Einschätzung ließe sich theoretisch vertiefen, was an dieser Stelle jedoch nicht geschehen soll. Stattdessen soll die weitere Klärung solcher Einwände mit der Frage nach der Wirksamkeit interreligiöser Bildung verbunden werden.

\section{Zur Wirksamkeit interreligiöser Bildung}

Im Folgenden soll zunächst ein Überblick zum Stand der Forschung hinsichtlich der Konzeptualisierung interreligiöser Bildung im Sinne von Kompetenzen sowie zu empirischen Befunden zum interreligiösen Lernen und dessen Wirksamkeit gegeben werden. In einem weiteren Schritt wird ein Forschungsprojekt dargestellt.

\subsection{Stand der Forschung}

\subsubsection{Konzeptualisierung interreligiöser Kompetenz}

Untersuchungen zur Wirksamkeit von Bildungsangeboten fragen heute in der Regel nach den dabei (nicht) erworbenen Kompetenzen. Diese Frage ist bei interreligiöser Bildung ebenfalls sinnvoll, auch wenn alternativ beispielsweise einfach nach dem Abbau von Vorurteilen gefragt werden könnte oder nach den (subjektiven) Wahrnehmungen der Schülerinnen und Schüler.

Welche Kompetenzmodelle sind in der Religionspädagogik verfügbar, und wie wird interreligiöse Bildung dabei aufgenommen? In den ersten Jahren der vor allem im Anschluss an die PISA-Studien in Gang gekommenen Kompetenzdiskussion wurde lebhaft über Kompetenzen und Kompetenzmodelle für den Religionsunterricht diskutiert (Disukssion: Rothgangel und Fischer 2004; Sajak 2012). Am Ende dieser 
Diskussion standen mehrere Vorschläge für Kompetenzmodelle, aber keines dieser Modelle konnte sich allgemein durchsetzen. Die in kirchlichen Stellungnahmen und staatlichen Vorgaben in den Bildungsplänen beschriebenen Kompetenzen sind insofern eher als politische Setzungen zu bezeichnen und erwachsen nicht aus einem theoretisch entwickelten Modell. Insbesondere aber fehlt es an einem auch empirisch validierten Modell, ähnlich wie bei anderen Fächern und Bildungsbereichen, die bei der Arbeit des IQB (Institut zur Qualitätsentwicklung im Bildungswesen) nicht berücksichtigt werden. Die den Bildungsplänen zugrunde gelegten Modelle eignen sich auch kaum für eine empirische Validierung, da sie eher als Anleitung von Praxis entworfen sind. Die einzige Ausnahme stellen hier zum einen die Modellbildungen einer erziehungswissenschaftlich-religionspädagogischen Berliner Forschungsgruppe um Dietrich Benner dar (Benner et al. 2011) und zum anderen die speziell auf interreligiöse Bildung bezogenen u.a. Tübinger Konzeptualisierungen zur interreligiösen Bildung (Schweitzer et al. 2017), in beiden Fällen auch verbundenen mit größeren empirischen Untersuchungen.

Das von der Berliner Forschungsgruppe für den Religionsunterricht vorgelegte Modell (später weitergeführt für den Ethikunterricht, Benner und Nikolova 2016) kommt mit drei Komponenten aus und ist insofern für empirische Validierungen besonders anschlussfähig: religionskundliche Kenntnisse, Deutungsfähigkeit, Partizipationsfähigkeit (Benner et al. 2011, S. 31 ff.). Die religionskundlichen Kenntnisse wurden zunächst nicht als Kompetenzkomponente aufgefasst, sondern als Voraussetzung von Deutungs- und Partizipationsfähigkeit. In der späteren Fassung des Modells für den Ethikunterricht werden solche Kenntnisse - konsequenterweise - ebenfalls als Kompetenz verstanden. Deutungsfähigkeit zielt auf ein weiterreichendes Verstehen und schließt insbesondere die Fähigkeit zur Perspektivenübernahme ein, in der dann in interreligiösen Zusammenhängen die eigentliche Zuspitzung entsprechender Herausforderungen für die Deutungsfähigkeit gesehen werden kann. Bei der Partizipationsfähigkeit, die sich empirisch nicht validieren ließ, geht es nicht etwa um die Beteiligung an religiösen Riten, sondern um die Fähigkeit, sich kompetent beispielsweise an religionsbezogenen Diskursen und Auseinandersetzungen in Politik und Öffentlichkeit zu beteiligen. Obwohl sich die Berliner Studie auf religiöse Kompetenz im Allgemeinen bezieht, enthält sie Anteile, die interreligiöse Zusammenhänge betreffen. Ein eigenes Modell zur interreligiösen Kompetenz wurde erst später sowie allein in theoretischer Hinsicht vorgelegt (Willems 2011).

Im Anschluss an dieses allgemeine Modell religiöser Kompetenz wurde insbesondere bei Tübinger Untersuchungen zum interreligiösen Lernen von drei Komponenten ausgegangen: Wissen, Perspektivenübernahme, Einstellungen (Schweitzer et al. 2017, S. 18 ff.). Das Wissen bezieht sich dabei auf verschiedene Religionen, ebenso die Fähigkeit zur Perspektivenübernahme. Einstellungen wurden ebenfalls eigens berücksichtigt, weil angesichts von Spannungen und Konflikten in der Gesellschaft Haltungen wie interreligiöse Offenheit und Toleranz unverzichtbar erscheinen.

Die drei genannten Kompetenzen (Wissen, Perspektivenübernahme, Einstellungen im Sinne der Offenheit) können als eine Art Konsens in der religionspädagogischen Diskussion gesehen werden. Hier gibt es zugleich zum Teil sehr komplexe, theologisch-theoretisch begründete Vorschläge für Kompetenzmodelle, die allerdings nicht auf empirische Anschlussfähigkeit zielen (s. etwa Schambeck 2013) 
oder von vornherein eher auf eine praxisorientierende Funktion hin angelegt sind (Meyer 2019). Bei manchen Modellen werden noch weitere Komponenten benannt etwa Wahrnehmen, Verstehen, Urteilen, Kommunizieren, Partizipieren, die als solche durchaus plausibel sind, aber kaum alle zugleich in ein empirisch validierbares Kompetenzmodell eingebracht werden können. Insofern erscheint es zumindest dann, wenn die Konzeptualisierung auch für eine empirische Validierung offen sein soll und sich der Frage nach der Wirksamkeit interreligiöser Bildung nicht entziehen will, empfehlenswert, sich auf die drei genannten Komponenten zu beschränken. Damit sind weitere, speziell auf andere Aspekte eingestellte Untersuchungen natürlich nicht ausgeschlossen. Es erscheint aber wenig wahrscheinlich, dass beispielsweise Wahrnehmungs- und Kommunikationsprozesse im interreligiösen Bereich unabhängig von einem entsprechenden Wissen, der Fähigkeit zur Perspektivenübernahme und offenen Haltungen funktionieren.

Im vorliegenden Zusammenhang ist darüber hinaus wichtig, dass diese Kompetenzen erkennbar nicht etwa auf eine Verfestigung religiöser Identitäten zielen. Kompetenzzuwächse würden vielmehr gerade bedeuten, dass ein Verstehen aus anderen Perspektiven möglich wird und die Offenheit im Blick auf religiöse Vielfalt wächst. Ebenso folgen sie nicht etwa einem einseitigen Verständnis von Integration, sondern zielen auf Toleranz und wechselseitige Anerkennung.

\subsubsection{Empirische Befunde im Überblick}

Während die theoretische Diskussion zu interreligiöser Bildung in Deutschland etwa 30 Jahre zurückreicht und international gesehen sogar mehr als 50 Jahre (besonders im United Kingdom, vgl. Copley 1997), sind empirische Untersuchungen, bei denen auch die Wirksamkeit entsprechender Bildungsangebote insbesondere im Rahmen des schulischen Religionsunterrichts in den Blick genommen wird, national und international eher selten geblieben. Dies erklärt sich zum einen aus dem insgesamt noch immer als sehr vorläufig zu bezeichnenden Stand der empirischen Forschung in der Religionspädagogik, insbesondere soweit es um Untersuchungen zu Unterrichtsprozessen und zur Wirksamkeit von Religionsunterricht geht (Schweitzer und Boschki 2018), zum anderen aber auch aus dem hohen Aufwand, den die für Wirksamkeitsuntersuchungen in der Regel erforderlichen Interventionsstudien mit sich bringen.

Allerdings erlauben zum Teil auch anders angelegte Studien zumindest erste Erkenntnisse. Das gilt beispielsweise für die Untersuchung der bereits erwähnten Berliner Forschungsgruppe, die den Stand der Ausprägung religiöser Kompetenz in Klasse 10 erhoben und dabei auch Aspekte interreligiöser Kompetenz einbezogen hat (Benner et al. 2011, S. $135 \mathrm{ff}$.). Da der Besuch von Regionsunterricht in Berlin nicht verpflichtend ist, stand hier eine Vergleichsgruppe zur Verfügung, die nicht an einem solchen Unterricht teilgenommen hatte. Die Befunde zeigen, dass - auch nach Kontrolle des Einflusses von Hintergrundfaktoren wie der religiösen Sozialisation die interreligiöse Kompetenz bei den Jugendlichen, die am Religionsunterricht teilgenommen hatten, deutlich stärker ausgeprägt war, als bei denen, auf die das nicht zutraf (S. 137). Zu ähnlichen Befunden kam auch eine in Österreich durchgeführte Untersuchung von Georg Ritzer (2010). Hier zeigte sich, „dass SchülerInnen, die 
an Ethikunterricht oder an Religionsunterricht teilnehmen, signifikant mehr über außerchristliche Weltreligionen wissen als SchülerInnen, die keines der beiden Fächer besuchen. Auch der Wissenszuwachs während eines Schuljahres ist bei SchülerInnen des RU/EU signifikant größer als bei AbmelderInnen vom RU, die an ihrer Schule nicht die Möglichkeit haben, an EU teilzunehmen“ (S. 279). Beim Umgang mit Pluralität, der bei dieser Studie ebenfalls als Kompetenz untersucht wurde, ließen sich allerdings keine entsprechenden Unterschiede feststellen. Es zeigte sich jedoch, dass mit der Fähigkeit zur Perspektivenübernahme auch der ,Einsatz für religiöse Toleranz" zunimmt und dass auch das Wissen über außerchristliche Weltreligionen tolerante Einstellungen unterstützt (S. 369). Gleichwohl bleibt das - im Verhältnis zu den erwähnten Befunden etwas widersprüchlich anmutende - Ergebnis, dass Werte oder Haltungen im Laufe eines Schuljahres durch den Religionsunterricht nicht in feststellbarer Weise verändert wurden (S. 372).

Ein entscheidender Nachteil der bislang genannten Untersuchungen besteht darin, dass sie den jeweils besuchten Unterricht als Blackbox behandeln. Erfasst wird lediglich der Besuch, nicht aber die Art und Weise, wie unterrichtet wurde, und auch nicht, was jeweils unterrichtet wurde. Insofern erlauben sie keine differenzielle Einschätzung unterschiedlicher didaktischer Strategien (auch wenn bei der Studie von Ritzer der Einfluss des sog. unterrichtlichen Binnengeschehens im Sinne der methodischen Ausgestaltung des Unterrichts kontrolliert wurde). Um hier genauere Aufschlüsse zu gewinnen, sind neben qualitativen Studien (vgl. etwa Gärtner und Bettin 2015; Kraml und Sejdini 2018), bei denen genaue Abschätzungen von Wirkungszusammenhängen naturgemäß schwierig bleiben, vor allem Designs mit Prä-Post-Messungen sowie Kontrollgruppen erforderlich sowie Samples, die auf das jeweilige Design abgestimmt sind.

Die erste größere Studie dieser Art zum interreligiösen Lernen kam aus den Niederlanden (Sterkens 2001). Die Untersuchung bezog sich auf 32 Grundschulklassen (S. 138). Gefragt wurde nach dem Erwerb von Wissen, der Entwicklung von Gefühlen sowie von Haltungen jeweils im Blick auf die eigene sowie eine andere religiöse Tradition (S. 126). Das Design schloss Prä- und Post-Tests ein sowie Experimentalund Kontrollgruppen (S. 138ff.). Als Treatment diente ein spezielles, auf interreligiöse Fragen eingestellte Curriculum (S. $131 \mathrm{ff}$.). Bei allen Untergruppen, die in Entsprechung zur Religionszugehörigkeit gebildet wurden, war eine Zunahme des Wissens festzustellen (S. 164). In affektiver Hinsicht nahmen sowohl bei der Experimental- wie bei der Kontrollgruppe negative Gefühle ab - allerdings mit Ausnahme muslimischer Schülerinnen und Schüler und deren negativen Gefühle im Blick auf die Fremdgruppe (S. $171 \mathrm{ff}$.). Zusammenfassend wird eine Wirksamkeit interreligiösen Lernens konstatiert, allerdings mit den genannten Einschränkungen (S. 183).

Weitere Interventionsstudien ergaben ein ähnliches Bild. Eine Veränderung von Haltungen durch den Religionsunterricht bzw. die Unterrichtseinheiten, die als Treatments fungierten, war nicht festzustellen (Schweitzer und Bucher 2020; Ziebertz 2010).

Auf die derzeit neueste Interventionsstudie zum „Interreligiösen Lernen durch Perspektivenübernahme“ (Schweitzer et al. 2017) soll im nächsten Abschnitt eingegangen werden. An dieser Stelle ist noch auf eine etwas anders ausgerichtete Untersuchung hinzuweisen, bei der die Frage nach dem Zusammenhang zwischen 
sozialer Ungleichheit und interreligiösen Lernen im Zentrum steht. In dieser Studie (Unser 2019) wurde der Umgang mit Aufgaben zum interreligiösen Lernen im Religionsunterricht untersucht. Geprüft werden sollte, wovon ein erfolgreicher Umgang mit solchen Aufgaben abhängig ist. Im Einzelnen erfasst wird der Einfluss von Religiosität, kulturellem Kapital, familiärem Hintergrund und Intentionalität beim Lernen. Die Studie kommt zu dem Ergebnis, dass die bisher im Religionsunterricht üblichen Formen interreligiösen Lernens dann besonders erfolgreich sind, wenn eine bestimmte Form der christlich-religiösen Sozialisation vorliegt. Auch Mädchen sind erfolgreicher bei diesen Aufgaben als Jungen. Insofern erzeuge ein solcher Unterricht soziale Ungleichheit bzw. verstärke er eine solche Ungleichheit weiter.

Zusammenfassend stellt sich der Stand der Forschung zu interreligiöser Bildung als eher vorläufig dar. Zahlreiche Aspekte und Fragen wurden noch nicht empirisch untersucht. Schon jetzt zeichnen sich allerdings Anfragen an die theoretischen Erwartungen $a b$, die für die Weiterentwicklung solcher Ansätze bedeutsam sind. Besonders deutlich ist dies für die Erwartung, dass der Religionsunterricht Veränderungen bei den Einstellungen der Schülerinnen und Schüler erzielen kann. Zugleich unterstützen die Befunde aber auch die Annahme, dass interreligiöse Bildung in anderen Hinsichten wirksam sein kann.

\subsection{Die Studie „Interreligiöses Lernen durch Perspektivenübernahme“6}

\subsubsection{Fragestellung, Hypothesen, Methode und Durchführung}

Untersucht werden sollte bei dieser von der Deutschen Forschungsgemeinschaft geförderten Studie ${ }^{1}$ die Wirksamkeit zweier verschiedener Treatments (Unterrichtseinheiten) bei verschiedenen Schülergruppen (ausführliche Darstellung: Schweitzer et al. 2017; die Darstellung im Folgenden stützt sich auf diese Veröffentlichung). Als Kriterien der Wirksamkeit wurden, der oben beschriebenen Konzeptualisierung folgend, Zuwächse beim Wissen und bei der Fähigkeit zur Perspektivenübernahme sowie Veränderungen bei den Einstellungen im Sinne von größerer Offenheit für andere Religionen festgelegt.

Die Untersuchung hatte das Design einer Interventionsstudie. Dabei wurde mit zwei verschiedenen Treatments in Gestalt interreligiös ausgerichteter Unterrichtseinheiten und entsprechend zwei Experimentalgruppen sowie einer Kontrollgruppe gearbeitet. Die Unterrichtseinheiten (UE) unterscheiden sich einerseits thematisch und andererseits in ihrer religionsdidaktischen Ausrichtung. Die eine Unterrichtseinheit hatte „Islamic Banking“ (ein Bankwesen ohne Zinsen) zum Thema, die andere „Religionen und Gewalt“. Da die Studie im Bereich des beruflichen Schulwesens durchgeführt wurde, u. a. in Bankklassen, wies die erste UE einen klaren Berufsbezug auf, während die andere UE keinen besonderen Lebenswelt- oder Berufsbezug einschloss. Beide UEs basieren auf Anforderungssituationen und folgen dem Bemühen, die interreligiöse Kompetenzentwicklung durch die Vermittlung interreligiösen Wissens und anhand von Übungen zur religionsbezogenen Perspektivenübernahme (etwa Rollenspiele) zu fördern.

\footnotetext{
${ }^{1}$ DFG-Zeichen: BI 674/4-1 und SCHW 568/17-1.
} 
Die Untersuchung der Wirksamkeit der Treatments erfolgte anhand eines Fragebogens, welcher zu drei Messzeitpunkten eingesetzt wurde, vor Beginn sowie nach der Durchführung der Einheiten und schließlich im Abstand etwa fünf Monate nach dem Unterricht. Der Fragebogen zielte auf die drei oben beschriebenen Kompetenzkomponenten religionsbezogenes Wissen, religionsbezogene Perspektivenübernahme und religionsbezogene Einstellungen. Der Fragebogen wurde in Anlehnung an die oben beschriebenen Studien neu entwickelt. Schließlich wurden diverse Hintergrundfaktoren erfasst.

Die Untersuchung ging hypothesengeleitet vor. Folgende Hypothesen wurden formuliert:

1. Beide Treatments haben eine positive Wirkung auf das religionsbezogene Wissen und die religionsbezogene Perspektivenübernahme.

2. Das berufsbezogene Treatment ,Islamic Banking“ bewirkt einen größeren Zuwachs des religionsbezogenen Wissens und der Fähigkeit zur religionsbezogenen Perspektivenübernahme als das nicht berufsorientierte Treatment (,Religionen und Gewalt").

3. Zwischen dem religionsbezogenen Wissen und der religionsbezogenen Perspektivenübernahme besteht ein positiver Zusammenhang (Wissen als notwendige, aber nicht hinreichende Voraussetzung religiöser Perspektivenübernahme).

4. Die Einstellungen der Schülerinnen und Schüler werden durch die Treatments nicht beeinflusst.

5. Die Wirkungen der beiden Treatments fallen bei muslimischen Schülerinnen und Schülern geringer aus als bei christlichen Schülerinnen und Schülern.

Die Studie wurde in Klassen des ersten und zweiten Ausbildungsjahrs von kaufmännischen Berufsschulen in Baden-Württemberg durchgeführt. Konkret ging es um die Ausbildungsgänge „Bankkauffrau/-mann“ und „Industriekauffrau/-mann“. Die finale Stichprobengröße lag bei $N=1105$ Schülerinnen und Schülern, die an allen drei Messzeitpunkten teilnahmen.

Die Auswertung erfolgte auf der Grundlage des beschriebenen Designs. So konnten ebenso die Unterschiede zwischen den beiden UEs wie auch die ausbildungsgangspezifischen Unterschiede konsequent berücksichtigt werden. Verglichen werden konnten zum einen jede der Experimentalgruppen mit der Kontrollgruppe sowie zum anderen die Experimentalgruppen miteinander. Die Größe der Stichprobe ließ mehrebenenanalytische Berechnungen (zur Nestung der Schülerinnen und Schüler in Klassen) zu, um beispielsweise den Einfluss unterschiedlicher Lehrkräfte zu kontrollieren.

Die drei Komponenten „religionsbezogenes Wissen“ (RWi) und „religionsbezogene Perspektivenübernahme“ (RPÜ) sowie ,religionsbezogene Einstellungen“ (RE) ließen sich in einer explorativen Faktorenanalyse mit den Daten des ersten Messzeitpunktes identifizieren (vgl., auch zum Folgenden, Losert 2017). Es konnten Skalen für das religionsbezogene Wissen (vier Faktoren) ( $\mathrm{t}_{1}$ : Cronbachs $\alpha$ zwischen 0,67 und 0,81 ) und für die religionsbezogene Perspektivenübernahme (zwei Faktoren) $\left(t_{1}: \alpha\right.$ zwischen 0,81 und 0,87) erstellt werden. Bei den religionsbezogenen Einstellungen wurden fünf Faktoren identifiziert ( $\mathrm{t}_{1}: \alpha$ zwischen 0,59 und 0,85). Der erste 
Faktor für Perspektivenübernahme verweist auf einen beruflichen Kontext, während der zweite sich als Perspektivenübernahme im Privatleben interpretieren lässt.

Vor der Faktorenanalyse wurde die Antwortkodierung und Skalierung der Aufgaben angepasst. Die Aufgaben zu RWi und RPÜ waren so erstellt worden, dass stets eine eindeutig richtige Antwort vorliegt. Die Aufgaben wurden entsprechend dichotomisiert und die Aufgabenschwierigkeit, d.h. der Anteil richtiger Antworten in der Stichprobe berechnet. Aufgaben mit mehr als $80 \%$ richtige Antworten in $t_{1}$ wurden aufgrund des damit einhergehenden Deckeneffekts bei $t_{2}$ und $t_{3}$ ausgeschieden, ebenso eine zu schwierige Aufgabe mit weniger als $8 \%$ richtige Antworten. $^{2}$

Zur Vorbereitung der Faktorenanalyse wurde eine Parallelanalyse (Horn 1965) zur Bestimmung der Anzahl zu extrahierender Faktoren unter Verwendung der für dichotome Antwortformate geeigneten tetrachorischen Korrelationsmatrix durchgeführt (Garrido et al. 2011, 2013). Die so bestimmte angemessene Anzahl an Faktoren wurde durch Hauptachsen-Faktorisierung (Principal-Axis-Factoring) extrahiert und mit dem Promax-Verfahren rotiert. Auf Grundlage der Pattern-Ladungsmatrix (vgl. z. B. Rencher und Christensen 2012; Rummel 1988) Aufgaben ausgeschlossen, deren Ladungsbetrag bei keinem der Faktoren $>0,30$ betrug. Eine erneute ParallelAnalyse stellte sicher, dass sich die Anzahl der zu extrahierenden Faktoren dadurch nicht verändert hatte.

Die Kompetenzen auf den Skalen zu RPÜ und RWi - für jeden Messzeitpunkt wurden unter Anwendung von Modellen der Item Response Theorie berechnet. Modellvergleiche zwischen 1PL-Modell (Raschmodell), 2PL-Modell (Birnbaummodell) und 3PL-Modell mittels Likelihood-Verhältnis-Tests ergaben für alle Skalen die Wahl des 2PL-Modells. Die Schätzungen für die Ausprägung der RE der Schülerinnen und Schüler sowie ihrer Religiosität erfolgte ebenfalls durch Modelle der Item Response Theorie. Die Likelihood-Verhältnis-Tests offenbarten, dass für alle Skalen das generalisierte Partial Credit Modell gegenüber dem Partial Credit Modell zu bevorzugen ist.

Auf dieser Grundlage wurden für jede Skala in Mehrebenenmodellen die oben genannten inhaltlichen Hypothesen überprüft. Die Kompetenz auf der jeweiligen Skala wurde dabei durch die festen Effekte von Messzeitpunkt (dummycodiert mit $\mathrm{t}_{1}$ als Referenzkategorie), Treatment, Religionszugehörigkeit und Ausbildungsgang - sowie je nach Modell eine oder mehrere Interaktionen zwischen diesen Variablen - vorhergesagt. Dann wurden Modellvergleiche (Likelihood-Quotienten-Tests) durchgeführt, die den inhaltlichen Hypothesen entsprachen. Die zufälligen Effekte wurden für jede Skala ebenfalls durch Modelltests festgelegt. Sie umfassten Random Intercepts (Baseline-Unterschiede) und Random Slopes (Lerneffekt-Unterschiede) auf Personen- und Klassenebene. Aus Raumgründen muss hier auf die detaillierte Darstellung der Methodik bei Losert (2017, S. 117 ff.) verwiesen werden.

\footnotetext{
2 Ich danke Martin Losert, der als Psychologe die Darstellung zur Methode durchgesehen und ergänzt hat.
} 


\subsubsection{Befunde}

Die Ergebnisse fallen für die verschiedenen Komponenten unterschiedlich aus:

- Für das religionsbezogene Wissen konnte in beiden Experimentalgruppen eine klare und stabile Kompetenzförderung durch die Unterrichtseinheiten nachgewiesen werden, die über die Wissenszuwächse in der Kontrollgruppe hinausging. Der Lerneffekt war auch noch mehrere Monate nach der Unterrichtsdurchführung vorhanden. Die Effekte betrugen in den meisten Fällen ca. eine Standardabweichung und können daher als große Effekte interpretiert werden.

- Bei der Fähigkeit zur Perspektivenübernahme im Privatleben waren ebenfalls einige stabile, mittelgroße Lerneffekte in Höhe von ca. einer halben Standardabweichung - im Vergleich zur Kontrollgruppe - zu verzeichnen. Im Ausbildungsgang der Industriefachklassen war bei beiden Unterrichtseinheiten eine dauerhafte Steigerung der Fähigkeit zu konstatieren. In Bankfachklassen war hingegen nur für die Einheit „Religionen und Gewalt“ eine langfristige Stärkung der Kompetenz zu verzeichnen. Bei der Fähigkeit zur Perspektivenübernahme im Beruf konnten keine zeitlich stabilen Lerneffekte nachgewiesen werden.

Hypothese 1 bestätigt sich damit im Blick auf das religionsbezogene Wissen, aber nur zum Teil für die Perspektivenübernahme.

- Im Blick auf die beiden Unterrichtseinheiten ergab sich, dass die Unterrichtseinheit „Islamic Banking“ weder für allgemeines religionsbezogenes Wissen noch für die Perspektivenübernahme zeitlich stabile, größere Kompetenzzuwächse erzielte als die Einheit „Religionen und Gewalt“. In den Bankfachklassen bewirkte, entgegen der Erwartungen im Projekt, die Einheit „Religionen und Gewalt“ einen zeitlich stabilen, größeren Kompetenzgewinn hinsichtlich der Perspektivenübernahme im Privatleben als die Einheit „Islamic Banking“.

Hypothese 2 konnte damit nicht bestätigt werden.

- Empirische Hinweise für den erwarteten Zusammenhang, dass religionsbezogenes Wissen eine Voraussetzung für religionsbezogene Perspektivenübernahme darstelle (im Sinne einer notwendigen, aber nicht hinreichenden Voraussetzung) fanden sich nur für die Perspektivenübernahme im Privatleben. Für die Perspektivenübernahme im Beruf ergab sich kein vergleichbarer Befund.

Hypothese 3 kann damit nur als teilweise bestätigt bezeichnet werden.

- Keine der beiden Unterrichtseinheiten bewirkte erkennbare Veränderungen bei den Einstellungen der Schülerinnen und Schüler.

Hypothese 4 kann somit bestätigt werden.

- Welche Wirksamkeit die Unterrichtseinheiten bei muslimischen Schülerinnen und Schülern erzielten, lässt sich aufgrund der geringen Stichprobengröße $(\mathrm{N}=50)$ nur 
schwer beurteilen. Beim religionsbezogenen Wissen ergab sich bei muslimischen Schülerinnen und Schülern ein vergleichsweise geringer Zugewinn, was eventuell auf einen Deckeneffekt zurückgeführt werden kann. Die Auszubildenden mit muslimischer Religionszugehörigkeit verfügten bei $t_{1}$ bereits über ein deutlich größeres Wissen (z-standardisierter Mittelwert $M=1,57$ ) als die christlichen Auszubildenden $(M=-0,07)$. Durch den Unterricht erhöhte sich das Wissen auch bei den muslimischen Schülerinnen und Schülern (um 0,57 Einheiten auf $M=2,14$ ), der Lernzuwachs war jedoch bei den christlichen Schülerinnen und Schülern noch größer (um 1,05 Einheiten auf $M=0,98$ ).

Hypothese 5 kann damit als teilweise bestätigt bezeichnet werden, aber mit dem deutlich einschränkenden Hinweis auf die geringe Stichprobengröße.

\subsubsection{Diskussion}

Für das Anliegen interreligiöser Bildung ist zunächst ermutigend, dass sich der untersuchte Unterricht zumindest in bestimmten Hinsichten als wirksam erwies. Insofern ist eine generelle Skepsis im Blick auf diese Bildungsaufgabe nicht begründet. Am klarsten fällt die Wirksamkeit beim religionsbezogenen Wissen aus. Dies entspricht einer Auffassung von Schule und Unterricht, die deren besondere Chance in der Erschließung geordneten Wissens sieht. Im Blick auf die Perspektivenübernahmefähigkeit, deren Stärkung in theoretischen Konzeptionen zum interreligiösen Lernen fast durchweg als wichtiges Ziel angesehen wird, ergibt sich kein abschließendes Bild. Zwar kann auch hier von einer - gewissen - Wirksamkeit des Unterrichts gesprochen werden, aber die Effekte sind zum Teil widersprüchlich und nicht flächendeckend. Dabei spielt auch die Frage eine Rolle, wie die Fähigkeit zur Perspektivenübernahme empirisch verlässlich erfasst werden kann. Auch bei anderen Studien (vgl. Losert et al. 2015; Merkt et al. 2014; Schweitzer und Bucher 2020) zeigten sich hier inkonsistente Ergebnisse. Dies verweist zugleich auf ein Problem für die Praxis in Schule und Religionsunterricht, da es in der Praxis kaum verlässlicher als in der Wissenschaft gelingen dürfte, wie von den Bildungsplänen vorausgesetzt einen entsprechenden Kompetenzzuwachs eindeutig zu identifizieren. In einem noch laufenden Anschlussprojekt ${ }^{3}$ wird deshalb ein anderer Zugang zur Messung der Fähigkeit zur Perspektivenübernahme verwendet, der sich insbesondere an die Untersuchungen von Milton J. Bennett (1986) anschließt. Ob sich daraus auch weitere Aufschlüsse im Blick auf den Zusammenhang zwischen religionsbezogenem Wissen und religionsbezogener Perspektivenübernahmefähigkeit ergeben, muss sich noch zeigen.

Angesichts der in der Gesellschaft stark wahrgenommenen Notwendigkeit, Einstellungen im Sinne von Toleranz, Anerkennung und wechselseitigem Respekt zu stärken, wirkt das Ausbleiben entsprechender Effekte in diesem Bereich besonders enttäuschend. Dieser Befund steht allerdings in Kontinuität zu den berichteten Befunden aus früheren Untersuchungen, weshalb auch die entsprechende Hypothese negativ formuliert war. Mitunter wurde bei Präsentationen im Blick auf diesen Be-

\footnotetext{
${ }^{3}$ DFG-Zeichen: BO 1747/11-1 und SCHW 568/23-1.
} 
fund als Erklärung vorgeschlagen, dass eine einzelne Unterrichtseinheit eben viel zu kurz sei, um Einstellungen zu verändern. Auf den ersten Blick erscheint dies plausibel, aber dagegen kann zugleich der Einwand erhoben werden, dass bei einer Intervention von kurzer Dauer zumindest kleine Effekte zu erwarten wären, wenn sich längerfristig größere Effekte einstellen sollen.

Religionsdidaktisch besonders überraschend ist, dass sich die stärker auf die Lebens- und Erfahrungswelt der einen Versuchsgruppe (Bank-Klassen) bezogene Unterrichtseinheit als weniger wirksam erwies als die allgemein thematische Einheit „Religionen und Gewalt“. Dies kann so wahrgenommen werden, dass dies dem weithin akzeptierten (religions-)didaktischen Prinzip der Subjektorientierung widerspricht. Auf jeden Fall kann darin ein Hinweis gesehen werden, dass das Prinzip der Subjektorientierung weiter präzisiert werden muss. Beispielsweise könnte es sein, dass es einer optimalen Ausprägung der Subjektorientierung bedarf, die dann von zu viel oder auch zu wenig Subjektorientierung zu unterscheiden wäre. Auch zu dieser Frage soll das oben genannte Nachfolgeprojekt weitere Aufschlüsse bieten.

Der Befund, dass die Zunahme religionsbezogene Wissens (zum Islam) bei den muslimischen Schülerinnen und Schülern geringer ausfiel als bei den übrigen Schülerinnen und Schülern, enthält interessante Implikationen im Blick auf die Frage, ob Religionsunterricht am besten im Klassenverband und also unabhängig von der Religionszugehörigkeit der Kinder und Jugendlichen erteilt werden soll. Auch wenn die muslimische Stichprobe der vorliegenden Untersuchung zu klein war für allgemeine Aussagen, lässt sich daraus die Frage ableiten, ob unterschiedliche Gruppen von Schülerinnen und Schülern nicht stärker von einem nach Konfessionen und Religionen differenzierten Angebote profitieren.

\section{Ausblick: Ergebnis und Aufgaben für die Weiterarbeit}

Eine Antwort auf grundsätzliche Einwände gegen interreligiöse Bildung und den Verdacht der Sinnlosigkeit wurde in diesem Beitrag vor allem im Blick auf die empirische Prüfung der Wirksamkeit entsprechender Bildungsangebote versucht. Offenbar lässt sich interreligiöse Bildung sinnvoll so konzeptualisieren, dass zum einen Kompetenzkomponenten ausgewiesen werden, die sich empirisch erfassen lassen. Dabei greift zum anderen weder der Vorwurf einer Religiösierung noch eines Othering, da entsprechende Kompetenzzuwächse nicht eine Verfestigung religiöser Identitäten bedeuten, sondern die Zunahme der Vertrautheit mit anderen Religionen, eine darauf bezogene Fähigkeit der Perspektivenübernahme sowie Offenheit für den anderen.

Die noch sehr überschaubare Reihe empirischer Untersuchungen zur Wirksamkeit interreligiöser Bildung im Religionsunterricht lässt insgesamt durchaus Effekte entsprechender Bildungsangebote erkennen. Am klarsten kann dies im Blick auf den Erwerb eines Wissens behauptet werden, das sich auf verschiedene Religionen bezieht. Das Bild bei der Perspektivenübernahmefähigkeit bleibt demgegenüber wesentlich unsicherer, was zugleich auf Messprobleme bei dieser Kompetenz hinweist. In dieser Hinsicht sind - für die Wissenschaft ebenso wie für die Praxis - weitere Entwicklungsaufgaben für die Identifikation dieser Kompetenz zu konstatieren. Der 
sich bei verschiedenen Untersuchungen wiederholende Befund, dass bei den Einstellungen keine Veränderungen erzielt werden konnten, wirft grundlegende Rückfragen im Blick auf entsprechende Erwartungen an den Unterricht auf. Offenbar muss in dieser Hinsicht über weitere Möglichkeiten wie beispielsweise Projekte oder Aktionen, Begegnungen und Kooperationen mit außerschulischen Partnern sowie über deren Wirksamkeit geforscht werden. Der theoretisch geforderte Effekt einer Verstärkung der Offenheit für andere Religionen konnte bei den Interventionsstudien bislang nicht belegt werden. Allerdings waren auch keine gegenläufigen Effekte etwa im Sinne einer zunehmenden Selbstabschließung zu beobachten.

Auch für interreligiöse Bildung gilt, dass sie nicht dann schon erfolgreich ist, wenn sie in der schulischen Praxis aufgenommen wird. Die Befundlage verweist vielmehr auf die Notwendigkeit didaktischer Präzisierungen, die über die Berufung auf generelle Prinzipien wie etwa Subjektorientierung hinausgehen. Dabei ist es eine offene Frage, wie sich ein solches didaktisches Prinzip theoretisch und empirisch genauer fassen lässt und wie es - beispielsweise im Sinne unterschiedlicher Grade der Ausprägung - weiter ausdifferenziert werden kann.

Ein abschließendes Urteil darüber, ob und in welchem Maße Angebote interreligiöser Bildung den zu Beginn genannten gesellschaftlichen Erfordernissen gerecht werden, lässt sich auf der Grundlage des bislang erreichten Forschungsstandes sowie der eigenen Befunde noch nicht formulieren. Immerhin geben die beobachteten Effekte aber doch Grund zu vorsichtigem Optimismus, der allerdings bei weiteren Untersuchungen auf die Probe gestellt werden sollte.

Förderung Parts of the research reported in this article were funded by the German Research Foundation (DFG BI 674/4-1 und SCHW 568/17-1.).

Funding Open Access funding enabled and organized by Projekt DEAL.

Open Access Dieser Artikel wird unter der Creative Commons Namensnennung 4.0 International Lizenz veröffentlicht, welche die Nutzung, Vervielfältigung, Bearbeitung, Verbreitung und Wiedergabe in jeglichem Medium und Format erlaubt, sofern Sie den/die ursprünglichen Autor(en) und die Quelle ordnungsgemäß nennen, einen Link zur Creative Commons Lizenz beifügen und angeben, ob Änderungen vorgenommen wurden.

Die in diesem Artikel enthaltenen Bilder und sonstiges Drittmaterial unterliegen ebenfalls der genannten Creative Commons Lizenz, sofern sich aus der Abbildungslegende nichts anderes ergibt. Sofern das betreffende Material nicht unter der genannten Creative Commons Lizenz steht und die betreffende Handlung nicht nach gesetzlichen Vorschriften erlaubt ist, ist für die oben aufgeführten Weiterverwendungen des Materials die Einwilligung des jeweiligen Rechteinhabers einzuholen.

Weitere Details zur Lizenz entnehmen Sie bitte der Lizenzinformation auf http://creativecommons.org/ licenses/by/4.0/deed.de.

Interessenkonflikt F. Schweitzer gibt an, dass kein Interessenkonflikt besteht.

\section{Literatur}

Auernheimer, G. (1990). Einführung in die interkulturelle Erziehung. Darmstadt: WBG.

Benner, D., \& Nikolova, R. (Hrsg.). (2016). Ethisch-moralische Kompetenz als Teil öffentlicher Bildung (Der Berliner Ansatz zur Konstruktion und Erhebung ethisch-moralischer Kompetenzen im öffentlichen Erziehungs- und Bildungssystem mit einem Ausblick auf Projekte zu ETiL-International). Paderborn: Schöningh. 
Benner, D., Schieder, R., Schluß, H., \& Willems, J. (Hrsg.). (2011). Religiöse Kompetenz als Teil öffentlicher Bildung. Versuch einer empirisch, bildungstheoretisch und religionspädagogisch ausgewiesenen Konstruktion religiöser Dimensionen und Anspruchsniveaus. Paderborn: Schöningh.

Bennett, M. (1986). A developmental approach to training for intercultural sensitivity. International Journal of Inercultural Relations, 10, 189-196.

Bohl, T., Budde, J., \& Rieger-Ladich, M. (Hrsg.). (2017). Umgang mit Heterogenität in Schule und Unterricht. Grundlagentheoretische Beiträge, empirische Befunde und didaktische Reflexionen. Bad Heilbrunn: Klinkhardt.

Copley, T. (1997). Teaching religion: fifty years of religious education in England and Wales. Exeter: University of Exeter Press.

Diehm, I. (2010). Religion ist im Spiel - oder virulent. Diskursive und interaktive Inszenierungen ethnischer Differenz. In C. Hunner-Kreisel \& S. Andresen (Hrsg.), Kindheit und Jugend in muslimischen Lebenswelten (S. 59-76). Wiesbaden: VS.

Dressler, B. (2003). Interreligiöses Lernen - alter Wein in neuen Schläuchen? Einwürfe in eine stagnierende Debatte. Zeitschrift für Pädagogik und Theologie, 55, 113-124.

EKD = Evangelische Kirche in Deutschland (Hrsg.). (2014). Religiöse Orientierung gewinnen. Evangelischer Religionsunterricht Beitrag zu einer pluralitätsfähigen Schule. Eine Denkschrift des Rates der EKD. Gütersloh: Gütersloher Verlagshaus.

Engebretson, K., de Souza, M., Durka, G., \& Gearon, L. (Hrsg.). (2010). International handbook of interreligious education (2. Aufl.). Dordrecht: Springer.

Garrido, L.E., Abad, F. J., \& Ponsoda, V. (2011). Performance of Velicer's minimum average partial factor retention method with categorical variables. Educational and Psychological Measurement, 71(3), 551-570.

Garrido, L.E., Abad, F. J., \& Ponsoda, V. (2013). A new look at Horn's Parallel Analysis with ordinal variables. Psychological Methods, 18(4), 454-474.

Gärtner, C., \& Bettin, N. (Hrsg.). (2015). Interreligiöses Lernen an außerschulischen Lernorten. Empirische Erkundungen zu didaktisch inszenierten Begegnungen mit dem Judentum. Berlin: LIT.

Hamburger, F. (2009). Abschied von der Interkulturellen Pädagogik. Plädoyer für einen Wandel sozialpädagogischer Konzepte. Weinheim: Juventa.

Horn, J.L. (1965). A rationale and test for the number of factors in factor analysis. Psychometrika, 30(2), 1789-1185.

Hull, J. M. (1996). A critique of Christian religionism in recent British education. In J. Astley \& L. J. Francis (Hrsg.), Christian theology and religious education: connections and contradictions (S. 140-165). London: SPCK.

Kraml, M., \& Sejdini, Z.Z. (Hrsg.). (2018). Interreligiöse Bildungsprozesse. Empirische Einblicke in Schul- und Hochschulkontexte. Stuttgart: Kohlhammer.

Krimmer, E. (2013). Evangelischer Religionsunterricht und reflektierte Toleranz. Aufgaben und Möglichkeiten religiöser Bildung im Pluralismus. Göttingen: V\&R.

Lähnemann, J. (1998). Evangelische Religionspädagogik in interreligiöser Perspektive. Göttingen: V\&R.

Langenhorst, G. (2016). Trialogische Religionspädagogik. Interreligiöses Lernen zwischen Judentum, Christentum und Islam. Freiburg: Herder.

Leimgruber, S. (2007). Interreligiöses Lernen. München: Kösel.

Lingen-Ali, U., \& Mecheril, P. (2016). Religion als soziale Deutungspraxis. Österreichisches religionspädagogisches Forum ÖRF, 24, 17-24.

Losert, M. (2017). Empirische Befunde zur interreligiösen Kompetenzentwicklung in Bank- und Industriefachklassen. In F. Schweitzer, M. Bräuer \& R. Boschki (Hrsg.), Interreligiöses Lernen durch Perspektivenübernahme. Eine empirische Untersuchung religionsdidaktischer Ansätze (S. 101-132). Münster: Waxmann.

Losert, M., Merkt, H., \& Schweitzer, F. (2015). In search of interreligious competence: an empirical study in the context of training caregivers through religious education. Journal of Empirical Theology, 28, 90-112.

Merkt, H., Schweitzer, F., \& Biesinger, A. (Hrsg.) (2014). Interreligiöse Kompetenz in der Pflege. Pädagogische Ansätze, theoretische Perspektiven und empirische Befunde. Münster: Waxmanmn.

Meyer, K. (2019). Grundlagen religiösen Lernens. Göttingen: V\&R.

Nipkow, K.E. (1998). Religionspädagogik im Pluralismus (Bildung in einer pluralen Welt, Bd. 2). Gütersloh: Gütersloher Verlagshaus.

Oelkers, J., Osterwalder, F., \& Tenorth, H.-E. (Hrsg.). (2003). Das verdrängte Erbe. Pädagogik im Kontext von Religion und Theologie. Weinheim: Dt. Studienverlag. 
Pant, H. A. (2016). Einführung in den Bildungsplan 2016 [Baden-Württemberg]. http://www.bildungsplaenebw.de/,Lde/LS/BP2016BW/ALLG/EINFUEHRUNG. Zugegriffen: 12. März 2021.

Prengel, A. (1993). Pädagogik der Vielfalt. Verschiedenheit und Gleichberechtigung in Interkultureller, Feministischer und Integrativer Pädagogik. Opladen: Leske + Budrich.

Radtke, F.-O. (2011). Kulturen sprechen nicht. Die Politik grenzüberschreitender Dialoge. Hamburg: Hamburger Edition.

Religionsmonitor (2015). Sonderauswertung Islam 2015. Die wichtigsten Ergebnisse im Überblick. Gütersloh: Bertelsmann.

Rencher, A.C., \& Christensen, W.F. (2012). Methods of multivariate analysis. Hoboken: Wiley.

Rickers, F. (2001). Art. Interreligiöses Lernen. In N. Mette \& F. Rickers (Hrsg.), Lexikon der Religionspädagogik (S. 874-881). Neukirchen-Vluyn: Neukirchener.

Ritzer, G. (2010). Interesse - Wissen - Toleranz - Sinn. Ausgewählte Kompetenzbereiche und deren Vermittlung im Religionsunterricht. Eine Längsschnittstudie. Wien: LIT.

Rothgangel, M., \& Fischer, D. (Hrsg.). (2004). Standards für religiöse Bildung. Zur Reformdiskussion in Schule und Lehrerbildung. Münster: LIT.

Rummel, R. J. (1988). Applied factor analysis. Evanston: Northwestern University Press.

Sajak, C.P. (Hrsg.). (2012). Religionsunterricht kompetenzorientiert. Beiträge aus fachdidaktischer Forschung. Paderborn: Schöningh.

Sajak, C.P. (2018). Interreligiöses Lernen. Darmstadt: WBG.

Schambeck, M. (2013). Interreligiöse Kompetenz. Basiswissen für Studium, Ausbildung und Beruf. Göttingen: $\mathrm{V} \& \mathrm{R}$.

Schweitzer, F. (2014). Interreligiöse Bildung. Religiöse Vielfalt als Herausforderung und Chance. Gütersloh: Gütersloher Verlagshaus.

Schweitzer, F., \& Boschki, R. (Hrsg.). (2018). Researching religious education. Classroom processes and outcomes. Münster: Waxmann.

Schweitzer, F., \& Bucher, I. (Hrsg.). (2020). Judentum und Islam im Religionsunterricht.Theoretische Analysen und empirische Befunde im Horizont interreligiösen Lernens. Münster: Waxmann.

Schweitzer, F., Englert, R., Schwab, U., \& Ziebertz, H.-G. (2002). Entwurf einer pluralitätsfähigen Religionspädagogik. Freiburg, Gütersloh: Herder \& Gütersloher Verlagshaus.

Schweitzer, F., Wissner, G., Bohner, A., Nowack, R., Gronover, M., \& Boschki, R. (2018). Jugend - Glaube - Religion. Eine Repräsentativstudie zu Jugendlichen im Religions- und Ethikunterricht. Münster: Waxmann.

Schweitzer, F., Bräuer, M., \& Boschki, R. (Hrsg.). (2017). Interreligiöses Lernen durch Perspektivenübernahme. Eine empirische Untersuchung religionsdidaktischer Ansätze. Münster: Waxmann.

Sterkens, C. (2001). Interreligious learning. The problem of interreligious dialogue in primary education. Leiden: Brill.

Unser, A. (2019). Social inequality and interreligious learning. An empirical analysis of students' agency to cope with interreligious learning tasks. Wien: LIT.

Willems, J. (2011). Interreligiöse Kompetenz. Theoretische Grundlagen - Konzeptualisierungen - Unterrichtsmethoden. Wiesbaden: VS.

Ziebertz, H.-G. (Hrsg.). (2010). Gender in Islam und Christentum. Theoretische und empirische Studien. Berlin: LIT. 